\title{
Medición del Trastorno por Estrés Postraumático (TEPT) en universitarios mexicanos
}

\author{
Sheila Adriana Mendoza Mojica, ${ }^{1}$ Octavio Márquez Mendoza, ${ }^{2}$ Rosalinda Guadarrama Guadarrama, ${ }^{2}$ \\ Luciana Esther Ramos Lira ${ }^{3}$
}

Artículo original

\section{SUMMARY}

Nowadays violence in different aspects and contexts affects Mexico in a worrying manner. Common traumatic experiences in some communities might configure grave mental health problems in the individuals, among them posttraumatic stress disorder (PTSD). Having scales that measure events susceptible to become traumatic as well as posttraumatic symptomatology would allow a fast assessment before formal diagnosis, to be mainly used in epidemiological studies that enable detecting the impact of these occurrences in vulnerable people and communities. The objective of the present study was to build a scale of events and symptoms associated to PTSD in a Mexican university population, in views to obtaining its reliability and validity. A total of 858 students from four public universities of Tejupilco, State of Mexi$\mathrm{co}$, Mexico, took part; they were between 18 and 25 years of age, and answered the scale in its entirety. The reliability index was obtained by means of Cronbach's alpha and the validity of the construct with factorial analysis. The most frequent event was the experience of sudden death of some relative and/or a close friend. The scale as a screening instrument obtained internal consistency indexes of $\alpha=0.95$ and the factorial analysis yielded five factors with a total variance of $45.15 \%$. Its design allows associating the symptoms of the previous year to a specific life event, valued by the same subject as susceptible to become traumatic, which permits its utilization on similar populations. The vulnerability of the young student population to suffer PTSD or any other sort of psychiatric disorder from experienced events was verified. Early detection could suggest general and specialized mental health services, accompanied by actions that recognize the value of university as one of the main social spaces for youths.

Key words: Measurement, mental health, PTSD, university students, trauma.

\section{RESUMEN}

Actualmente la violencia en diferentes ámbitos y contextos afecta de manera preocupante a México. Las experiencias traumáticas, comunes en algunas comunidades, pueden llegar a configurar problemas graves de salud mental en los individuos, entre ellos se cuenta el Trastorno por Estrés Postraumático (TEPT). El tener a la mano escalas que midan tanto los eventos susceptibles de ser traumáticos como la sintomatología postraumática, permitiría una evaluación rápida antes del diagnóstico formal y su utilización, principalmente en estudios epidemiológicos que permitan detectar el impacto de dichos sucesos en las personas y las comunidades. El objetivo del presente estudio consistió en la construcción de una escala de sucesos y síntomas asociados al TEPT en población mexicana universitaria, con el fin de obtener su confiabilidad y validez.

Participaron 858 estudiantes de cuatro universidades públicas de Tejupilco, Estado de México. La muestra fue de 669 jóvenes de ambos sexos con edades de 18 a 25 años, quienes contestaron la escala en su totalidad. El índice de confiabilidad se obtuvo con una alfa de Cronbach y la validez de constructo mediante el análisis factorial.

El suceso más frecuentemente encontrado fue la experiencia de muerte repentina de algún familiar $y / o$ amigo cercano. La escala, como instrumento de tamizaje, obtuvo índices convenientes para ser aplicada en poblaciones similares. La consistencia interna resultó con una $\alpha=.95$ y el análisis factorial arrojó cinco factores con una varianza total de $45.15 \%$.

Su diseño permite asociar los síntomas del último año a un suceso de vida específico, valorado por el mismo sujeto, como susceptible a ser traumático. Así, se detectó vulnerabilidad en la población juvenil estudiantil a padecer el TEPT o algún otro tipo de trastorno psiquiátrico derivado de los sucesos vividos.

La identificación precoz podría sugerir la creación de servicios generales y especializados en salud mental, acompañados de acciones que reconozcan el valor de la Universidad como uno de los principales espacios sociales para jóvenes.

Palabras clave: Medición, salud mental, TEPT, universitarios, trauma.

Centro Universitario Temascaltepec, Extensión Tejupilco. Universidad Autónoma del Estado de México.

Centro de Investigación en Ciencias Médicas. Universidad Autónoma del Estado de México.

Dirección de Investigaciones Epidemiológicas y Psicosociales. Instituto Nacional de Psiquiatría Ramón de la Fuente Muñiz.

Correspondencia: Sheila Adriana Mendoza Mojica. Domicilio Conocido, Rincón de Aguirre, 51425, Tejupilco, Estado de México. E-mail: shememo06@ yahoo.com.mx

Recibido primera versión: 12 de octubre de 2011. Segunda versión: 10 de junio de 2013. Tercera versión: 24 de septiembre de 2013. Aceptado: 14 de octubre de 2013. 


\section{INTRODUCCIÓN}

La presencia de eventos traumáticos en integrantes de algunas comunidades, aun siendo comunes, generan reacciones posteriores que, dada la variabilidad de circunstancias, afectarán la salud mental del individuo.

No existe la definición unívoca de una experiencia de este tipo debido a sus características, por lo que debe tomarse en cuenta la forma en cómo el individuo la vive y experimenta; es decir, la relación particular que se establece entre ambos. ${ }^{1}$

Horowitz et al., ${ }^{2}$ retomando las investigaciones de Holmes y Rahe realizadas en 1967 sobre la respuesta humana al estrés, lograron considerar algunos sucesos de vida como traumáticos o susceptibles de serlo desde su valoración.

Así, en 1979 diseñaron la Escala de Impacto del Estresor (EIE) con el fin de evaluar el malestar subjetivo que acompaña y sigue a las experiencias traumáticas y/o estresantes. El instrumento está constituido por dos factores: intrusión y evitación, y por medio de 15 reactivos midieron su intensidad y frecuencia. La aplicación se llevó a cabo en dos muestras distintas obteniéndose índices de confiabilidad mediante el alfa de Cronbach. En general, para la primera muestra fue de .86; para el factor de intrusión, de .78 y para el de evitación, .82. En la segunda muestra fue de .87 general, para el factor de intrusión 89 y de evitación .79 .

Con estas cifras, el instrumento mostró pertinencia para detectar sintomatología característica del TEPT a partir de la medición de eventos susceptibles de experimentarse traumáticamente. En 1980 se introdujo por primera vez el TEPT en la tercera edición del Manual Diagnóstico y Estadístico de los Trastornos Mentales (DSM) editado por la Asociación Americana de Psiquiatría (APA). La experiencia de sucesos traumáticos de los veteranos de guerra y de mujeres víctimas de abuso sexual, delimitaron los grupos de síntomas, vigentes hasta la actualidad. ${ }^{3}$

Las ediciones posteriores han presentado cambios principalmente relacionados con la conceptualización del evento traumático. Para la última versión revisada del Manual, en 2005 (DSM-IV-TR), los criterios establecidos para diagnosticar el TEPT quedaron de la siguiente manera: ${ }^{4}$

A. La persona ha estado expuesta a un acontecimiento traumático en el que han existido 1 y 2 :

1. la persona ha experimentado, presenciado o le han explicado uno (o más) acontecimientos caracterizados por muertes o amenazas para su integridad física o la de los demás.

2. la persona ha respondido con un temor, una desesperanza o un horror intensos.

B. El cuadro sintomático característico, secundario a la exposición al intenso trauma, debe incluir la presencia de reexperimentación persistente del acontecimiento traumático.

C. Evitación persistente a los estímulos asociados a él y embotamiento de la capacidad de respuesta del individuo.

D. Síntomas persistentes de activación (arousal).
Para las versiones siguientes se ha sugerido replantear algunos de estos criterios, principalmente los relacionados al suceso (A). Los argumentos comunes refieren la ambigüedad en la conceptualización del evento traumático según los distintos grupos de la población (edad, sexo, raza); según la diversidad cultural que influye en la percepción subjetiva de la gravedad para ser valorado como susceptible de vivirse traumáticamente, principalmente porque es un proceso posterior a la ocurrencia física del suceso estresante..$^{5-7}$

El tiempo e intensidad, así como las características individuales y comunitarias del sujeto, son cruciales para desarrollar dicho trastorno. Los factores demográficos y socioeconómicos intervienen también como riesgos de exposición a experiencias traumáticas y al TEPT posterior, teniendo un impacto en la salud mental del individuo. ${ }^{8}$

Las encuestas de salud mental internacionales dejan ver la prevalencia existente, demostrando sintomatología del TEPT y comorbilidad en sus sociedades. En los Estados Unidos, mediante The National Comorbidity Survey Replication $^{9}$ en 2007, con 5692 ciudadanos mayores de 18 años, se observó una prevalencia de vida del TEPT de $6.8 \%$ y para el último año de vida de $3.5 \%$. Al respecto, The National Survey of Adolescents, con 4023 adolescentes, encontró 3.7\% en hombres y $6.3 \%$ en mujeres para seis meses de prevalencia.

En población colombiana, la prevalencia general del TEPT en 2004, fue de $0.2 \%$, en tanto que $1.8 \%$ lo padeció alguna vez en su vida. Para la población desplazada por eventos violentos, en 2008, se encontró que $1.4 \%$ padecía síntomas del TEPT actuales y 4.22\% los presentó alguna vez en su ciclo vital. Ello permitió observar que ciertos eventos violentos como amenazas, muertes y combates (vividos de forma directa o indirecta) generan una mayor sintomatología del TEPT. ${ }^{10}$

En Chile, durante el 2009, Pérez et al. encontraron una prevalencia de $4.4 \%$ en una muestra representativa nacional. ${ }^{11}$ Los autores destacaron la importancia de desarrollar estudios epidemiológicos transculturales del TEPT debido a las diferencias encontradas en países de raíces similares, atribuidas a factores socio-económicos como la magnitud de desigualdad entre ricos y pobres, violencia, crimen y pobreza.

En 2005, un estudio realizado en una zona urbana de México resaltó que $68 \%$ de su población adulta había estado expuesta, al menos alguna vez en su vida, a un suceso estresante asociado al TEPT. Los sucesos más frecuentes fueron: persecución o acoso, violación, secuestro, abuso sexual y haber sido víctima de violencia por parte de sus padres. Respecto al trastorno, la prevalencia total fue de $1.45 \% .{ }^{12}$

Así mismo, la aplicación de The Composite International Diagnostic Interview (CIDI) con adolescentes, realizada en la Zona Metropolitana de la Ciudad de México, obtuvo, para la sección del TEPT, un $68.9 \%$ de prevalencia de vida donde al menos una vez habían experimentado un suceso traumático asociado. Los más comunes fueron: muerte súbita e inesperada de un familiar, testigos de violencia domés- 
tica y estar involucrado en un accidente grave. ${ }^{13}$ Un $28.2 \%$ de los jóvenes mencionaron haber vivido dos o tres eventos traumáticos, mientras que $13 \%$ reportaron cuatro o más.

Estas prevalencias se han obtenido por medio de entrevistas semiestructuradas, sin embargo los instrumentos de cribado o tamizaje permiten una medición rápida antes de un diagnóstico formal. Suelen ser de gran utilidad para estudios epidemiológicos o en poblaciones vulnerables, variando su uso según los objetivos y la población estudiada. ${ }^{14}$

A pesar de ello, en 2003, ${ }^{15}$ de veinticinco instrumentos, sólo seis estaban adaptados y validados al idioma español. Además, las poblaciones en las que se habían validado correspondían a personas con un suceso específico y/o bajo algún tratamiento médico o psicológico.

$\mathrm{Su}$ aplicación en otros contextos se encuentra al margen de los fines para los cuales fueron creados. Sin embargo, su estructura muestra índices de confiabilidad y validez. La Escala de Trauma de Davidson (DTS) mide la gravedad y frecuencia de 17 síntomas en individuos que han experimentado un trauma. Posee una consistencia interna de $\alpha=.99$ general. Para la subescala de frecuencia, $\alpha=.97$ y para la de gravedad, $\alpha=.98$. Respecto a su estructura factorial, la gravedad explica el $24.79 \%$ del total de varianza en sujetos sin TEPT; con TEPT, resultaron seis factores con una varianza explicada de $26.91 \%$. Para la frecuencia, los ítems fueron de intrusión, evitación y embotamiento. ${ }^{16}$

La aplicación de ésta en población española obtuvo una consistencia interna de $\alpha=.89$ general. La subescala del criterio B (reexperimentación) $\alpha=.83$, para el criterio C (evitación), $\alpha=.74$ y el criterio D (hiperactivación), $\alpha=.76$. Para este estudio no se realizó análisis factorial y la población estudiada fue en personas con y sin TEPT. ${ }^{17}$

Por otra parte, la Escala del Impacto del Estresor (EIE) representa uno de los instrumentos de autoinforme utilizado con mayor frecuencia para evaluar la sintomatología postraumática en adultos. Una adaptación de esta escala, realizada por Weiss et al. en $1996,{ }^{18}$ consideró el grupo de síntomas de hiperactivación (incluyendo enojo e irritabilidad), creando así la Escala Revisada del Impacto del Estresor (EIE-R) de 22 ítems. En dos muestras de personas víctimas de terremotos, en Estados Unidos, la confiabilidad representó para la primera muestra de 429 sujetos un $\alpha=.87$ para el factor de intrusión, $\alpha=.85$ el de evitación y $\alpha=.79$ el de hiperactivación. La segunda muestra fue de 197 personas, con $\alpha=.91$ para intrusión, $\alpha=.84$ evitación y $\alpha=.90$ de hiperactivación.

En 2003 se realizó un meta-análisis con 40 estudios respecto a la confiabilidad y validez de la EIE, encontrándose una media de consistencia interna para el factor intrusión de $\alpha=.86$ (.72-.92). Para el factor evitación, $\alpha=.82$ (.65-.90). Los resultados finales sugieren la presencia de un tercer factor a partir de la división de evitación. ${ }^{19}$

Considerando que en México, uno de cada cinco individuos presenta en su vida al menos un trastorno mental que se inicia en la mayoría de las veces entre los 19 y los 20 años de edad (siendo los de ansiedad más frecuentes y cróni$\cos ),{ }^{20}$ se planteó como objetivo el construir un instrumento de cribado para la población universitaria mexicana, con un diseño capaz de detectar sucesos de vida específicos de esta comunidad, así como la síntomatología actual asociada al TEPT y otros trastornos comórbidos, obteniéndose índices de confiabilidad y de validez pertinentes para ser utilizada en poblaciones similares.

\section{MATERIAL Y MÉTODOS}

Los participantes del estudio fueron 858 jóvenes de ambos sexos, inscritos en cuatro universidades públicas de la ciudad de Tejupilco, Estado de México, previa autorización de los directivos. Los sujetos accedieron contestar la escala de manera voluntaria a partir del consentimiento informado.

La muestra fue no probabilística por conveniencia, conformada por 669 (78\%) estudiantes de ambos sexos de entre 18 a 25 años, quienes al contestar la lista de sucesos vividos eligieron el más impactante, considerando que aún en la actualidad les perturbaba, describiéndolo y contestando completamente el apartado de sintomatología a partir de dicho suceso.

Las características del diseño de la escala fueron escogidas a partir de la revisión de instrumentos de cribado para el TEPT ${ }^{14,15,17}$ retomando la distribución de reactivos por factores según los criterios diagnósticos del DSM. Sin embargo, el instrumento resultó diferente dado que la redacción de reactivos fue contextualmente apropiada a la concepción del TEPT en la actualidad y a la población universitaria mexicana, lo cual se consideró mediante su revisión por parte de cinco expertos mexicanos en estudios clínicos y epidemiológicos de este trastorno. Además, se llevó a cabo una aplicación piloto para mejorar la redacción de los reactivos y precisar las instrucciones, el tiempo empleado y la disponibilidad de ser contestado. Se proporcionó un consentimiento informado aprobado por el Comité de Ética e Investigación del Centro de Investigación en Ciencias Médicas de la UAEMéx.

Para la aplicación definitiva, la escala quedó conformada por dos apartados: El primero es una lista de 32 sucesos considerados comúnmente como traumáticos, pudiendo generar síntomas de TEPT. Al frente se ubicaron casillas para marcar su ocurrencia: de vida y en los últimos doce meses. Al final, los sujetos eligieron el suceso de la lista que había impactado en su vida lo suficiente como para recordarlo en la actualidad de la misma forma, considerándolo amenazante para su integridad personal (criterio A). Sucesivamente lo describieron de manera general y señalaron la gravedad percibida en una escala del 1 al 10.

El segundo apartado se conformó por 65 reactivos referentes a la reexperimentación, evitación (alejamiento y em- 
Cuadro 1. Distribución de ocurrencia de sucesos susceptibles de ser traumáticos

\begin{tabular}{lrr}
\hline \multicolumn{1}{c}{ Sucesos traumáticos } & Frecuencia & $\%$ \\
\hline 1 Muerte repentina de algún familiar y/o & 163 & 24.4 \\
amigo cercano & 112 & 16.7 \\
2 Accidentes & 74 & 11.1 \\
3 Abuso físico & 66 & 9.9 \\
4 Amenaza y/o experiencia traumática a otros & 60 & 9.0 \\
5 Ser amenazado y/o vigilado & 51 & 7.6 \\
6 Estar a punto de ahogarse & 47 & 7.0 \\
7 Ser testigo de alguna atrocidad humana & 33 & 4.9 \\
8 Abuso sexual & 25 & 3.7 \\
9 Estar en medio de una guerra & 14 & 2.1 \\
10 Hospitalización & 9 & 1.3 \\
11 Desastres (naturales y/o humanos) & 7 & 1.0 \\
12 Haber herido o matado a alguien & 5 & .7 \\
13 Haber sufrido graves quemaduras & 2 & .3 \\
14 Haber sido rehén, secuestrado y/o torturado & 1 & .1 \\
15 Haber estado refugiado en otro país & 669 & 100.0 \\
\hline Total &
\end{tabular}

botamiento emocional) e hiperactivación, correspondientes a los criterios B, C y D del mismo manual en manifestaciones sociales, cognitivas, físicas y emocionales. La frecuencia de dichos síntomas se delimitó para los últimos doce meses, a partir del suceso previamente descrito aunque ocurrido años anteriores. Las respuestas se plantearon en una escala tipo Likert donde 1 fue nunca y 5 diariamente (anexo 1).

La estadística descriptiva se utilizó para obtener las frecuencias de sexo, edad, área de estudio y municipio de residencia. Respecto al suceso elegido: tipo de suceso (según el DSM-IV-TR) y edad de ocurrencia. Para considerar un suceso como traumático fue necesario previamente realizar un análisis cualitativo de la descripción generada por los sujetos. Las categorías fueron: descripción correspondiente al suceso elegido, manifestación de haber sentido amenazada su vida y/o integridad personal o la de alguien cercano, especificación de la edad de ocurrencia y determinar si lo habían vivido, testificado o se los habían contado.

La confiabilidad de los reactivos se obtuvo con el método de consistencia interna de todos los reactivos, mediante el coeficiente alfa de Cronbach usado por reactivo para calificarse de forma individual a lo largo de valores determinados. ${ }^{21}$

Respecto a la validez, se consideró la de constructo, obtenida por medio del método de análisis factorial, con la extracción de componentes principales y obteniendo la varianza total explicada con rotación varimax. Así mismo se extrajo el valor alfa para cada factor obtenido. Los procesos estadísticos se llevaron a cabo con el paquete estadístico SPSS versión 17.

\section{RESULTADOS}

De los 669 jóvenes que respondieron ambos apartados de la Escala, 53\% fueron mujeres y $47 \%$ hombres. La edad pre-
Cuadro 2. Carga factorial por reactivo en la conformación de factores

\begin{tabular}{|c|c|c|}
\hline Factor & Reactivos & Carga factorial \\
\hline 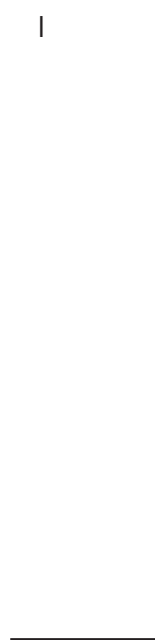 & $\begin{array}{l}13 \\
16 \\
17 \\
20 \\
29 \\
31 \\
32 \\
35 \\
41 \\
42 \\
45 \\
46 \\
47 \\
50 \\
51 \\
58 \\
64 \\
65\end{array}$ & $\begin{array}{l}.584 \\
.620 \\
.600 \\
.623 \\
.450 \\
.738 \\
.663 \\
.455 \\
.664 \\
.558 \\
.546 \\
.640 \\
.423 \\
.598 \\
.629 \\
.713 \\
.568 \\
.600\end{array}$ \\
\hline$\|$ & $\begin{array}{l}25 \\
26 \\
36 \\
38 \\
40 \\
43 \\
49 \\
52 \\
53 \\
54 \\
55 \\
56 \\
57 \\
59 \\
60 \\
61 \\
62\end{array}$ & $\begin{array}{l}.496 \\
.420 \\
.405 \\
.562 \\
.463 \\
.474 \\
.465 \\
.567 \\
.658 \\
.572 \\
.601 \\
.522 \\
.528 \\
.654 \\
.418 \\
.502 \\
.454\end{array}$ \\
\hline III & $\begin{array}{r}4 \\
5 \\
8 \\
10 \\
22 \\
24 \\
28 \\
30 \\
33 \\
34 \\
37 \\
48\end{array}$ & $\begin{array}{l}.542 \\
.581 \\
.580 \\
.525 \\
.486 \\
.420 \\
.515 \\
.481 \\
.633 \\
.603 \\
.592 \\
.563\end{array}$ \\
\hline IV & $\begin{array}{l}14 \\
15 \\
18 \\
23 \\
44\end{array}$ & $\begin{array}{l}.470 \\
.482 \\
.539 \\
.525 \\
.437\end{array}$ \\
\hline $\mathrm{V}$ & $\begin{array}{r}1 \\
2 \\
3 \\
7 \\
27 \\
63\end{array}$ & $\begin{array}{l}.634 \\
.518 \\
.528 \\
.501 \\
.418 \\
.462\end{array}$ \\
\hline $\begin{array}{l}\text { Reactivos } \\
\text { eliminados }\end{array}$ & $\begin{array}{c}6,9,11,12 \\
19,21 \text { y } 39\end{array}$ & $<.40$ \\
\hline
\end{tabular}


sentó una media de 19.94, el rango con mayor frecuencia fue de 18 a 20 años. Las áreas correspondientes a la carrera estudiada fueron: económico-administrativa (47\%), humanística-social (35\%) y físico-matemática (18\%).

Respecto a su lugar de residencia, 53\% manifestó vivir en el municipio de Tejupilco, seguidos en frecuencia por los municipios aledaños de Amatepec y Luvianos con un 7\% cada uno. El 33\% restante manifestó vivir en municipios diversos del mismo Estado de México y, en menor porcentaje, en Estados como Michoacán, Oaxaca y Distrito Federal.

En el cuadro 1 se presenta la distribución de ocurrencia de los sucesos considerados susceptibles de configurarse como traumáticos en la muestra. Los 32 sucesos se agruparon en 15 según la similitud de las características descritas.

Respecto a la edad de ocurrencia, la media fue de 17.59 años. El 60\% manifestó haberlo vivido entre los 15 y 19 años. El 26\%, de los cuatro a los 12 años y 14\%, de los 20 a los 25. Según el tipo de suceso, el $73.1 \%$ vivió directamente el suceso, $17 \%$ lo testificó y a $9.9 \%$ se lo contaron. La gravedad percibida resultó con una media de 6.95, siendo 10 el puntaje más frecuente.

La confiabilidad se obtuvo mediante el análisis de fiabilidad con el método de consistencia interna, realizando la correlación entre reactivos, obteniéndose un alfa de Cronbach general de .96 para los 65 reactivos.

La validez de constructo se realizó por medio del método de análisis factorial, con rotación varimax de Kaiser para la solución de matrices, obteniéndose 13 factores. Debido a la agrupación de reactivos se realizó nuevamente el análisis factorial para cinco factores, donde se consideró estaría la mayoría de ellos. Así, se obtuvo la carga factorial de 58 reactivos con valores superiores a .40 según el método por extracción, agrupándose en el factor donde obtuvieron un puntaje mayor (cuadro 2).

Estos cinco factores permitieron caracterizar la sintomatología del TEPT en la muestra según los criterios diagnósticos: Evitación de tipo embotamiento emocional (18), Reexperimentación de tipo fisiológico (17), Evitación de tipo alejamiento (12), Hiperactivación (5) y Reexperimentación de tipo cognitivo (6). Los factores explicaron una varianza total de $45.15 \%$ (cuadro 3).

Cuadro 3. Análisis factorial de la escala de trastorno por estrés postraumático en universitarios mexicanos

\begin{tabular}{clccc}
\hline Factor & Nombre & $\begin{array}{l}\text { Valor } \\
\text { eigen }\end{array}$ & Varianza & $\begin{array}{c}\text { Varianza } \\
\text { acumulada }\end{array}$ \\
\hline I & $\begin{array}{l}\text { Evitación de tipo } \\
\text { embotamiento emocional }\end{array}$ & 8.618 & 13.258 & 13.258 \\
II & $\begin{array}{l}\text { Reexperimentación } \\
\text { de tipo fisiológica }\end{array}$ & 6.961 & 10.709 & 23.968 \\
III & $\begin{array}{l}\text { Evitación de tipo alejamiento } \\
\text { IV }\end{array}$ & 6.600 & 10.154 & 34.121 \\
Viperactivación & 3.667 & 5.641 & 39.763 \\
& $\begin{array}{l}\text { Reexperimentación } \\
\text { de tipo cognitiva }\end{array}$ & 3.502 & 5.388 & $\mathbf{4 5 . 1 5 0}$ \\
\hline
\end{tabular}

Cuadro 4. Consistencia interna de los factores obtenidos para la escala de trastorno por estrés postraumático en universitarios mexicanos

\begin{tabular}{ccccc}
\hline Factor & $\begin{array}{c}\text { Alfa de } \\
\text { Cronbach }\end{array}$ & $\begin{array}{c}\text { Número de } \\
\text { elementos }\end{array}$ & Media & $\begin{array}{c}\text { Desviación } \\
\text { típica }\end{array}$ \\
\hline I & .919 & 18 & 25.06 & 9.527 \\
II & .911 & 17 & 24.62 & 8.932 \\
III & .864 & 12 & 22.33 & 8.783 \\
IV & .705 & 5 & 6.36 & 2.206 \\
V & .769 & 6 & 10.13 & 3.663 \\
General & $\mathbf{. 9 5 7}$ & 58 & 88.51 & 27.820 \\
\hline
\end{tabular}

Posteriormente se obtuvo la consistencia interna general de los 58 reactivos, resultando un alfa de .957 y por factor, los puntajes obtenidos fueron superiores a .70 (cuadro 4).

\section{DISCUSIÓN}

La prevalencia de sucesos traumáticos asociados al desarrollo del TEPT, considerados impactantes en su integridad física y emocional para esta población (78\%), fue superior a lo reportado por Medina-Mora et al..$^{12}$ en población urbana durante el 2005 (68\%). En ambos, el suceso más prevalente lo representó la experiencia de muerte repentina de algún familiar y/o amigo cercano, en la muestra fue de $24.4 \%$. El porcentaje restante lo representaron sucesos que coincidieron con lo reportado por Orozco et al. en $2008^{13}$ en población adolescente: alta presencia de accidentes de diversa índole y abuso físico (incluyendo la violencia doméstica). La población juvenil estudiantil participante de este estudio, estuvo expuesta durante su infancia y adolescencia en su mayoría, a actos de violencia en sus distintas manifestaciones, ${ }^{22}$ percibiéndolos amenazantes para la integridad y continuidad de su vida.

Respecto a la sintomatología del TEPT, la escala posee las propiedades psicométricas de un instrumento de tamizaje con índices de confiabilidad y validez convenientes para ser aplicada en poblaciones similares, procurando la detección oportuna y atención especializada posterior de cualquier trastorno mental asociado a estos sucesos.

La confiabilidad mostró una alta consistencia entre reactivos, con un alfa de .95 , siendo superior a la encontrada en población española (172 estudiantes universitarios) a los cuales se les aplicó la DTS ${ }^{16}$ donde la consistencia interna fue de $\alpha=.89$ en su totalidad. También resultó superior a la media general de $\alpha=.86$ reportada en un metanálisis de 40 estudios de la EIE $^{19}$ y de $\alpha=.87$ para la EIE-R, ${ }^{18}$ en una muestra de 429 personas. Y fue más cercana a la obtenida en la Escala de Gravedad de Síntomas del TEPT en 638 adultos jóvenes españoles, $\alpha=.92 .{ }^{23}$

Así mismo, la validez de constructo mostró la evidencia de las ventajas del diseño y construcción de la escala en universitarios, permitiendo asociar los síntomas a un suceso específico en la valoración del sujeto inmerso en un contexto de situaciones sociales de violencia. La región sur del Estado 
de México colinda con los Estados de Guerrero, Michoacán y Morelos, donde se registra el mayor índice delictivo con afectación severa, ${ }^{24}$ los acontecimientos que comparten generan una percepción de inseguridad en las personas debido a sucesos anteriores como victimización familiar y personal, crímenes con violencia y delitos sobre la propiedad en su entidad. ${ }^{25}$ Así mismo, Tejupilco ha sido escenario de la lucha entre cárteles por el control de las zonas productoras de drogas sintéticas y el trasiego de precursores químicos. ${ }^{26}$

La dinámica demográfica conduce a la emigración de comunidades limítrofes en los Estados de Michoacán, Morelos, Guerrero y México hacia ciudades como Tejupilco, generando mayor presión social por la demanda de empleos, educación y salud.

El análisis factorial representó una varianza total explicada de $45.15 \%$. Considerando .70 la puntuación límite para aceptar la prueba como confiable, ${ }^{27}$ la extracción de ellos permitió enunciarlos a partir del grupo de síntomas referidos por el DSM-IV-TR. ${ }^{4}$

Las diferencias encontradas pueden deberse al tamaño de la muestra y al número de reactivos, ya que entre más grande sean, los estimados de confiabilidad serán más cercanos a $1 .^{21}$ Así mismo, las consideraciones para el suceso susceptible de experimentarse traumáticamente varían. En este estudio se consideró pertinente caracterizarlo especificando el tipo, ocurrencia y valoración subjetiva del mismo, contemplando que el TEPT en la actualidad es un trastorno de ansiedad con manifestaciones clínicas más complejas.

El primer factor obtenido se denominó: "Evitación de tipo embotamiento emocional" como un factor específico del grupo de síntomas de evitación denominados en la EIE como aplanamiento emocional, insensibilidad/adormecimiento emocional o "emotional numbing". ${ }^{19}$ En ambas escalas, los ítems se diferencian claramente de los relacionados a la Evitación de tipo alejamiento.

Contrario a ello, en la EIE-R, ${ }^{28}$ validada en idioma español con 1078 adultos jóvenes españoles que no informaron haber vivido situación traumática alguna, se encontraron dos factores aislados: intrusión/hiperactivación y evitación sin posibilidad de dividir este último en dos: embotamiento y aislamiento.

Este grupo de síntomas distintivos del Estrés Postraumático ha sido referido por Foa et al., ${ }^{29}$ quienes resaltan que tiene relación con la prevalencia de vida del suceso. Los estudiantes manifestaron la presencia de esta sintomatología durante el último año, pero relacionado con la vivencia de un suceso experimentado durante su vida, constatando la vulnerabilidad para desarrollar un TEPT en esta población. Además, el afrontamiento de tipo desadaptativo por parte del sujeto puede llevarlo a comportamientos disfuncionales ante las amenazas posteriores ${ }^{30}$ teniendo impacto en sus relaciones sociales en general.

Su utilidad puede ampliarse ya que el TEPT no es exclusivo de las vivencias de acontecimientos traumáticos; la depresión mayor y el trastorno distímico son algunos de los padecimientos más frecuentemente asociados a este trastorno. ${ }^{31}$ Existe la posibilidad de incrementar la utilidad mediante el diseño de otros reactivos similares en aplicaciones futuras, principalmente en los factores de Hiperactivación y Reexperimentación de tipo cognitivo donde se encontraron los valores alfa más bajos.

Según la Encuesta Nacional de Epidemiología Psiquiátrica, en México, ${ }^{20}$ en 2003, solamente uno de cada 10 sujetos con algún trastorno mental recibía atención general, mientras que para la especializada, sólo la recibían dos de cada 30 sujetos con dos o más trastornos mentales. Además, uno de cada cinco individuos presentó al menos un trastorno mental en un momento de su vida.

Por otra parte, según la agenda para el DSM-5, el estudio de dicho trastorno plantea a desafíos significativos debido a la marcada predominancia del patrón occidental, por lo que para la siguiente edición se propone un enfoque de diagnóstico cultural. ${ }^{32}$ El grupo de edad, la diversidad cultural y la presencia de síntomas a partir del suceso ocurrido en varios de los participantes de este estudio muchos años antes, resalta la necesidad de considerar los criterios diagnósticos del TEPT. ${ }^{5-7}$ Por esto la escala representa una medición de la salud mental en México acorde al contexto de jóvenes mexicanos inmersos en un medio caracterizado por el incremento de la pobreza y el distanciamiento entre las posibilidades económicas y culturales de los distintos grupos sociales donde la violencia social puede incrementarse. ${ }^{33}$

Las Instituciones de Educación Superior en ciudades mexicanas representan un espacio ante esta realidad, ofreciendo servicios de calidad para formar jóvenes con elementos humanistas y culturales para que una vez que egresen puedan contribuir al desarrollo del país con la perspectiva de una sociedad global, armónica y solidaria. ${ }^{34}$

El cumplimiento de este objetivo resulta un reto, sin embargo estos estudios pueden coadyuvar mediante la identificación de los factores implicados en la salud mental juvenil, contemplando a futuro intervenciones que incluyan además de aspectos biológicos, los cognitivos, los conductuales $\mathrm{y}$, principalmente, los sociales.

\section{AGRADECIMIENTOS}

A las siguientes instituciones y personas: Consejo Nacional de Ciencia y Tecnología (CONACYT). Consejo Mexiquense de Ciencia y Tecnología (COMECYT). Centro de Investigación en Ciencias Médicas (CICMED), UAEMéx. Facultad de Medicina, UAEMéx. Centro Universitario UAEMéx Temascaltepec y a su Extensión Tejupilco. Universidad Tecnológica del Sur del Estado de México (UTSEM). Universidad Pedagógica Nacional, subsede Tejupilco y Universidad Mexiquense del Bicentenario, sede Tejupilco. A los doctores Rebeca Robles García, Benjamín Domínguez Trejo, Francisco Páez Agraz; a la maestra Iris Rubí Velasco Monroy; a los licenciados: Cuitláhuac Santiago, Nohemí Legorreta Ávalos y José de Jesús López Flores. 


\section{REFERENCIAS}

1. Sosa CD y Capafóns J. Estrés postraumático. España: Síntesis; 2005.

2. Horowitz M, Wilner N, Alvarez W. Impact of Event Scale: a measure of subjective stress. Psychosom Med 1979;41:209-218.

3. Bobes-García J, Bousoño-García M, Calcedo-Barba A, González-GarcíaPortilla MP. Trastorno de estrés postraumático. Barcelona: Masson; 2000.

4. American Psychiatric Association. Trastornos de ansiedad en el Manual Diagnóstico y Estadístico de los Trastornos Mentales. (DSM-IVTR) texto revisado. Barcelona: Masson; 2005.

5. Palacios L, Heinze G. Trastorno del estrés postraumático: una revisión del tema (segunda parte). Salud Mental 2002;25:61-71.

6. Brewin CR, Lanius RA, Novac A, Schnyder U et al. Reformulating PTSD for DSM-V: Life after Criterion A. J Trauma Stress 2009;22:366-373.

7. Kilpatrick DG, Resnick HS, Acierno R. Should PTSD Criterion A be retained? J Trauma Stress 2009;22:374-383.

8. Hidalgo RB, Davidson JR. Posttraumatic stress disorder: epidemiology and health-realted considerations. J Clin Psychiatry 2000;61:5-13.

9. Gradus JL. Epidemiology of PTSD. En: National Center for PTSD (United States Department of Veterans Affairs) 2007. Disponible en: http://www.ptsd.va.gov/professional/pages/epidemiological-factsptsd.asp, consultado enero 2010.

10. Echenique C, Medina LM, Medina A, Ramírez A. Prevalencia por trastorno del estrés postraumático en población desplazada por violencia, en proceso de restablecimiento en Sincelejo. Psicología desde Caribe, Universidad del Norte 2008;21:122-135.

11. Pérez-Benítez CI, Vicente B, Zlotnikc C, Kohn R et al. Estudio epidemiológico de sucesos traumáticos, trastorno de estrés post-traumático y otros trastornos psiquiátricos en una muestra representativa de Chile. Salud Mental 2009;32:145-153.

12. Medina-Mora Icaza ME, Borges-Guimaraes G, Lara C, Ramos-Lira L et al. Prevalencia de sucesos violentos y de trastorno por estrés postraumático en la población mexicana. Salud Pública Mex 2005;47:08-22.

13. Orozco R, Borges G, Benjet C, Medina-Mora ME et al. Traumatic life events and posttraumatic stress disorder among Mexican adolescents: results from a survey. Salud Pública Mex 2008;50(supl 1):s29-s37.

14. Bobes-García J, G-Portilla MP, Bascarán-Fernández MT, Saíz-Martínez PA et al. Banco de instrumentos básicos para la práctica de la psiquiatría clínica. Tercera edición. España: Ars Médica; 2004.

15. Crespo M, Gómez MM. Propuesta de un inventario para la evaluación y diagnóstico del Trastorno de Estrés Postraumático. Psicopatología Clínica, Legal y Forense 2003;3:41-57.

16. Villafañe A, Milanesio MS, Marcellino CM, Amodei C. La evaluación del Trastorno por Estrés Postraumático: aproximación a las propiedades psicométricas de la escala de trauma de Davidson. Evaluar 2003;julio:80-93.

17. Bobes J, Calcedo-Barba A, García M, Francois M et al. Evaluación de las propiedades psicométricas de la versión española de cinco cuestionarios para la evaluación del trastorno de estrés postraumático. Actas Esp Psiquiatr 2000;28:207-218.
18. Weiss DS, Marmar Ch. Propiedades psicométricas de la versión española de la Escala Revisada de Impacto del Estresor (EIE-R). Análisis Modificación Conducta 2001;27:581-604.

19. Sundin EC, Horowitz MJ. Impact of event scale: psychometric properties. Br J Psychiatry 2002;180:205-209.

20. Medina-Mora ME, Borges G, Lara C, Benjet C et al. Prevalencia de trastornos mentales y uso de servicios: resultados de la Encuesta Nacional de Epidemiología Psiquiátrica en México. Salud Mental 2003;26:1-16.

21. Cohen RJ, Swerdick ME. Confiabilidad en pruebas y evaluación psicológicas. Cuarta edición. México: Mc Graw Hill; 2001.

22. Baker C, Norris F, Dayna M, Diaz V et al. Violence and PTSD in Mexico, gender and regional differences. Soc Psychiatry Psychiatr Epidemiol 2005;40:519-528.

23. Echeburúa E, Decorral P, Amor PJ, Zubizarreta I et al. Escala de gravedad de síntomas del trastorno de estrés postraumático: propiedades psicométricas. Análisis Modificación Conducta 1997; 23:503-526.

24. Centro de Investigación para el Desarrollo AC (CIDAC), México 2013. Ocho delitos, primer índice delictivo CIDAC. Disponible en: www. cidac.org.

25. Notimex. El Universal Estados, lunes 05 de mayo de 2008. Disponible en: http://www.eluniversal.com.mx/notas/504261.html.

26. Castillo G (2012). Límites de Edomex, Guerrero y Michoacan, nueva zona de disputa entre grupos del narco. Periódico La Jornada, domingo 28 de octubre de 2012, p. 12. Disponible en: http://www.jornada. unam.mx/2012/10/28/politica/012n1pol

27. Kerlinger F, Lee H. Confiabilidad. En: Investigación del comportamiento. Métodos de investigación en Ciencias sociales. Cuarta edición. México: Mc. Graw Hill; 2006.

28. Báguena MJ, Villarroya E, Beleña A, Díaz A et al. Propiedades psicométricas de la versión española de la Escala Revisada de Impacto del Estresor (EIE-R). Análisis Modificación Conducta 2001;27:581-604.

29. Foa EB, Rothbaum BO. Estrés postrumático (EPT). México: Trillas; 2008.

30. Domínguez B, Pennebaker W, Olvera Y. Estrés postrumático (EPT). México: Trillas; 2008.

31. Vallejo-Ruiloba J, Gastó-Ferrer C, Cardoner-Álvarez N, Catalán-Campos R. Comorbilidad de los trastornos afectivos. España: Ars Médica; 2002.

32. Alarcón R, Bell C, Kirmayer L, Lin KM et al. Más allá de los tópicos. Agenda de Investigación sobre Cultura y Diagnóstico Psiquiátrico. En: Agenda de investigación para el DSM-V. Kupfer DJ, First MB y Regier DA (eds.). España: Masson; 2004.

33. González-Pérez GJ, Vega-López MG, Vega-López A, Muñoz-De la Torre A et al. Homicidios de adolescentes en México, 1979-2005: evolución y variaciones sociodemográficas. Papeles de población. México: Universidad Autónoma del Estado de México; 2009; 15:109-139.

34. Asociación Nacional de Universidades e Instituciones de Educación Superior, 2010. Propuestas para el desarrollo de la educación superior. En: Educación superior en el Siglo XXI, líneas estratégicas de desarrollo. Disponible en: http://www.anuies.mx/servicios/d_estrategicos/documentos_estrategicos/21/index.html, consultado en enero 2011.

Artículo sin conflicto de intereses 


\section{ANEXO 1 \\ UNIVERSIDAD AUTÓNOMA DEL ESTADO DE MÉXICO}

Centro de Investigación en Ciencias Médicas

\section{Escala para Estrés Postraumático en universitarios mexicanos}

El objetivo de este cuestionario es detectar sucesos y características de uno de ellos que haya sido peligroso para tu estabilidad o la de alguien cercano. Así mismo, las reacciones de dicho suceso durante el último año. Tu participación es valiosa ya que ayudará al desarrollo posterior de estrategias enfocadas a la promoción de la salud. Toda la información será manejada confidencialmente, por favor sé lo más sincero/a posible.

Fecha de nacimiento:

Edad:

Fecha de hoy:

Sexo: $\square$ Mujer $\square$ Hombre

Lugar de residencia:

Carrera:

Nivel:

En la siguiente lista de sucesos marca con una "X" el recuadro que represente tu situación especificando la edad en la que ocurrió. Si ha ocurrido durante el último año, además márcalo en el recuadro de enfrente.

\begin{tabular}{|c|c|c|c|c|c|c|}
\hline \multirow[b]{2}{*}{ Número } & \multirow[b]{2}{*}{ Sucesos } & \multicolumn{3}{|c|}{$\begin{array}{l}\text { Alguna vez } \\
\text { en la vida }\end{array}$} & \multicolumn{2}{|c|}{$\begin{array}{l}\text { Últimos } 12 \\
\text { meses }\end{array}$} \\
\hline & & No & Sí & Edad & No & Si \\
\hline 1 & Haber estado presente en un desastre natural como un huracán, terremoto o inundaciones devastadoras & & & & & \\
\hline 2 & Haber estado involucrado/a en un desastre causado por el ser humano & & & & & \\
\hline 3 & Haber tenido una enfermedad que te causara heridas graves o incluso poder llegar a morir & & & & & \\
\hline 4 & Haber sido asaltado/a o amenazado/a con un arma & & & & & \\
\hline 5 & Haber sido perseguido/a o vigilado/a para hacerte daño & & & & & \\
\hline 6 & $\begin{array}{l}\text { Haber estado expuesto/a a un tóxico o sustancia química peligrosa que pudiera provocarte daños graves } \\
\text { o la muerte }\end{array}$ & & & & & \\
\hline 7 & $\begin{array}{l}\text { Haberte visto involucrado/a en un accidente de vehículos de motor que te hubiera podido causar la muerte } \\
\text { o haberte dejado lesiones graves }\end{array}$ & & & & & \\
\hline 8 & $\begin{array}{l}\text { Haber tenido algún accidente de otro tipo que hubiera podido causarte la muerte o daños de gravedad } \\
\text { incluso en la escuela o trabajo }\end{array}$ & & & & & \\
\hline 9 & Haber estado a punto de ahogarte & & & & & \\
\hline 10 & Haber sufrido graves quemaduras & & & & & \\
\hline 11 & Haber recibido una paliza por algún familiar (no incluye a parejas o ex parejas) & & & & & \\
\hline 12 & Haber recibido una paliza por parte de una pareja o ex pareja & & & & & \\
\hline 13 & Haber recibido una paliza por parte de otra persona & & & & & \\
\hline 14 & Haber sido herido, cortado, rasguñado o sangrado por parte de algún padre o familiar cercano & & & & & \\
\hline 15 & Haber sido obligado/a a tener relaciones sexuales con alguien en contra de tu voluntad & & & & & \\
\hline 16 & $\begin{array}{l}\text { Haber sido obligado/a a tocar los genitales a alguien o haber sido tocado/a en tus genitales contra tu } \\
\text { voluntad; o haber sido tocado/a sexualmente contra tu voluntad, o de forma inapropiada }\end{array}$ & & & & & \\
\hline 17 & Haber participado en alguna guerra como miembro del ejército o de una organización no militar & & & & & \\
\hline 18 & Haber sido secuestrado/a & & & & & \\
\hline 19 & $\begin{array}{l}\text { Haber sido miembro de las fuerzas de paz, o colaborador/a humanitario/a en una zona en guerra o en } \\
\text { un lugar donde hubiera una situación de terror entre la población, debido a conflictos políticos, raciales, } \\
\text { religiosos o de cualquier otro tipo }\end{array}$ & & & & & \\
\hline 20 & Haber sido torturado/a & & & & & \\
\hline 21 & $\begin{array}{l}\text { Haber estado como civil desarmado/a en un lugar donde había una guerra, una revolución, un golpe de } \\
\text { estado militar o una invasión }\end{array}$ & & & & & \\
\hline 22 & $\begin{array}{l}\text { Haber vivido como civil en un lugar donde había una situación de terror entre la población por motivos } \\
\text { políticos, raciales, religiosos o de cualquier otro tipo }\end{array}$ & & & & & \\
\hline 23 & $\begin{array}{l}\text { Muerte de algún familiar o amigo/a muy cercano/a de forma repentina o inesperada (por ejemplo, en un } \\
\text { accidente, asesinato, suicidio o por un ataque al corazón a una edad temprana) }\end{array}$ & & & & & \\
\hline
\end{tabular}




\begin{tabular}{|c|c|c|c|c|c|c|}
\hline \multirow[b]{2}{*}{ Número } & \multirow[b]{2}{*}{ Sucesos } & \multicolumn{3}{|c|}{$\begin{array}{l}\text { Alguna vez } \\
\text { en la vida }\end{array}$} & \multicolumn{2}{|c|}{$\begin{array}{c}\text { Últimos } 12 \\
\text { meses }\end{array}$} \\
\hline & & No & Sí & Edad & No & Si \\
\hline 24 & Haber hecho algo que provocara accidentalmente heridas graves o muerte a alguna persona & & & & & \\
\hline 25 & Haber herido gravemente, torturado o matado a alguien a propósito & & & & & \\
\hline 26 & $\begin{array}{l}\text { Haber estado en situación de refugiado (tener que abandonar tu país de origen y refugiarte en el extranjero } \\
\text { para escapar del peligro o de la ejecución) }\end{array}$ & & & & & \\
\hline 27 & Haber sido testigo de golpizas entre familiares cerca de ti & & & & & \\
\hline 28 & $\begin{array}{l}\text { Haber visto o escuchado a alguien ser herido gravemente o a alguien que estaba siendo asesinado; o haber } \\
\text { visto inesperadamente un cadáver }\end{array}$ & & & & & \\
\hline 29 & Haber sido testigo de atrocidades o matanzas, por ejemplo mutilaciones o asesinatos en masa & & & & & \\
\hline 30 & Amenaza seria o daño a un miembro de tu familia o amigo/a íntimo & & & & & \\
\hline 31 & Experiencia traumática de algún familiar o amigo/a cercano/a como secuestro, violación o tortura & & & & & \\
\hline 32 & $\begin{array}{l}\text { ¿े Hay alguna otra situación que hubiera podido causarte la muerte o lesiones graves que no esté mencionada } \\
\text { en esta lista? Favor de especificar: }\end{array}$ & & & & & \\
\hline
\end{tabular}

Escoge el suceso que consideres más impactante, que aun después de ocurrido afectara tu bienestar y seguridad lo suficiente para comentarlo. Escribe el número al que se refiere.

a) Número de suceso

b) ¿Qué ocurrió?

c) ¿Qué edad tenías?

d) ¿Cuándo?

e) ¿Dónde?

f) ¿Con quién/es (familiares, amigos, etc.) ?

g) ¿Hubo víctimas mortales?

¿Cómo calificas la gravedad de lo ocurrido? Encierra el número que consideres representativo.

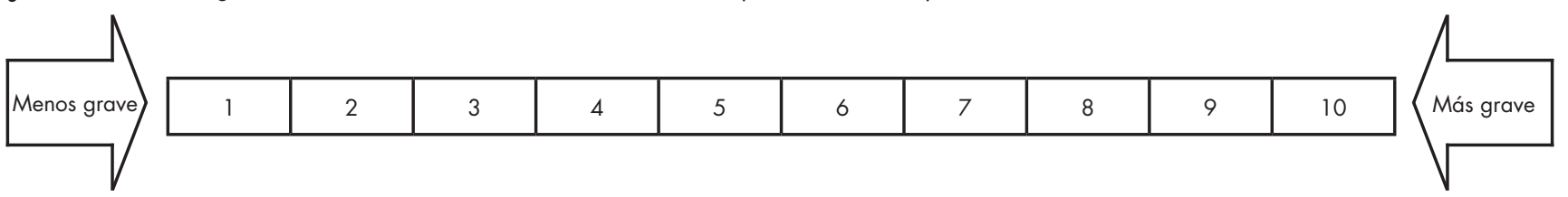


A partir del suceso anteriormente descrito responde si en el último año te han ocurrido las sensaciones, emociones, pensamientos y/o comportamientos siguientes. Marca con una "X" el recuadro de la derecha correspondiente usando las siguientes opciones de respuestas.

\begin{tabular}{|c|c|c|c|c|}
\hline Nunca & A veces & Varias veces & Varias veces & A diario \\
al memana & $\mathbf{4}$ & $\mathbf{5}$ \\
\hline
\end{tabular}

En los últimos doce meses qué tan frecuente te ha ocurrido...

\begin{tabular}{|c|c|c|c|c|c|c|}
\hline \multirow{2}{*}{$\frac{\text { Número }}{1}$} & \multirow{2}{*}{\begin{tabular}{|l|} 
Reacciones \\
Tener pesadillas
\end{tabular}} & \multicolumn{5}{|c|}{ Frecuencia } \\
\hline & & 1 & 2 & 3 & 4 & 5 \\
\hline 2 & Pensamientos desagradables & 1 & 2 & 3 & 4 & 5 \\
\hline 3 & Desgaste físico cuando pienso en ello & 1 & 2 & 3 & 4 & 5 \\
\hline 4 & Procurar no recordarlo & 1 & 2 & 3 & 4 & 5 \\
\hline 5 & Alejarme de personas que me lo recuerdan & 1 & 2 & 3 & 4 & 5 \\
\hline 6 & Exagerar en revisar a las personas que recientemente conozco & 1 & 2 & 3 & 4 & 5 \\
\hline 7 & Dificultad para concentrarme en actividades escolares & 1 & 2 & 3 & 4 & 5 \\
\hline 8 & Enojarme fácilmente & & & & & \\
\hline 9 & Actuar sin pensar porque me asusto fácilmente & 1 & 2 & 3 & 4 & 5 \\
\hline 10 & Temor a que vuelva a ocurrir & 1 & 2 & 3 & 4 & 5 \\
\hline 11 & Sentir dolor en el estómago y/o diarrea cuando tengo imágenes de lo que pasó & 1 & 2 & 3 & 4 & 5 \\
\hline 12 & Encontrar objetos relacionados & 1 & 2 & 3 & 4 & 5 \\
\hline 13 & Sentirme aislado/a del resto de la gente & 1 & 2 & 3 & 4 & 5 \\
\hline 14 & No asistir a festejos que me lo recuerden & 1 & 2 & 3 & 4 & 5 \\
\hline 15 & Sentirme enfermo/a cuando mis amigos quieren que hagamos actividades relacionadas & 1 & 2 & 3 & 4 & 5 \\
\hline 16 & Creer que el futuro no tiene nada bueno para mí & 1 & 2 & 3 & 4 & 5 \\
\hline 17 & Dificultad para sentir afecto por los demás & 1 & 2 & 3 & 4 & 5 \\
\hline 18 & Temblores, mareos y/o nauseas al recordarlo & 1 & 2 & 3 & 4 & 5 \\
\hline 19 & Observar desesperadamente los acontecimientos que ocurren cerca de mí & 1 & 2 & 3 & 4 & 5 \\
\hline 20 & Dejarme de importar una calificación aprobatoria en la escuela & 1 & 2 & 3 & 4 & 5 \\
\hline 21 & Sentir emociones rápidamente & 1 & 2 & 3 & 4 & 5 \\
\hline 22 & Desviar las conversaciones relacionadas & 1 & 2 & 3 & 4 & 5 \\
\hline 23 & Tener sudoración excesiva al recordarlo & 1 & 2 & 3 & 4 & 5 \\
\hline 24 & Inquietarme ante cualquier ruido familiar & 1 & 2 & 3 & 4 & 5 \\
\hline 25 & Sentir las mismas emociones que cuando ocurrió & 1 & 2 & 3 & 4 & 5 \\
\hline 26 & Vivir situaciones idénticas & 1 & 2 & 3 & 4 & 5 \\
\hline 27 & Dificultad para dormir & 1 & 2 & 3 & 4 & 5 \\
\hline 28 & Esforzarme en no recordarlo durante los eventos sociales & 1 & 2 & 3 & 4 & 5 \\
\hline 29 & Procurar no hacer nuevas amistades & 1 & 2 & 3 & 4 & 5 \\
\hline 30 & Alejarme inmediatamente de lugares parecidos & 1 & 2 & 3 & 4 & 5 \\
\hline 31 & Creer que el rumbo que tome mi vida no tiene importancia & 1 & 2 & 3 & 4 & 5 \\
\hline 32 & Perder interés de participar en actividades escolares & 1 & 2 & 3 & 4 & 5 \\
\hline 33 & Esforzarme en actuar como si nunca hubiera ocurrido & 1 & 2 & 3 & 4 & 5 \\
\hline 34 & Estar alerta inmediatamente ante cualquier situación similar & 1 & 2 & 3 & 4 & 5 \\
\hline 35 & Irritarme fácilmente & 1 & 2 & 3 & 4 & 5 \\
\hline 36 & Tener más rápidos los latidos del corazón cuando pienso en lo acontecido & 1 & 2 & 3 & 4 & 5 \\
\hline 37 & Intentar quitarme los recuerdos de la memoria & 1 & 2 & 3 & 4 & 5 \\
\hline 38 & Despertar asustado después de soñar que nuevamente ocurre & 1 & 2 & 3 & 4 & 5 \\
\hline 39 & Dejar de asistir a la escuela por estar pensando en ello & 1 & 2 & 3 & 4 & 5 \\
\hline 40 & Despertar cansado por recordarlo & 1 & 2 & 3 & 4 & 5 \\
\hline
\end{tabular}


A partir del suceso anteriormente descrito responde si en el último año te han ocurrido las sensaciones, emociones, pensamientos y/o comportamientos siguientes. Marca con una "X" el recuadro de la derecha correspondiente usando las siguientes opciones de respuestas.

\begin{tabular}{|c|c|c|c|c|}
\hline $\begin{array}{c}\text { Nunca } \\
1\end{array}$ & $\begin{array}{c}\text { A veces } \\
2\end{array}$ & $\begin{array}{c}\text { Varias veces } \\
\text { al mes } \\
\mathbf{3}\end{array}$ & $\begin{array}{c}\text { Varias veces } \\
\text { a la semana } \\
4\end{array}$ & $\begin{array}{c}\text { A diario } \\
5\end{array}$ \\
\hline
\end{tabular}

En los últimos doce meses qué tan frecuente te ha ocurrido...

\begin{tabular}{|c|c|c|c|c|c|c|}
\hline Número & Reacciones & \multicolumn{5}{|c|}{ Frecuencia } \\
\hline 41 & Creer que es mejor permanecer alejado de los demás & 1 & 2 & 3 & 4 & 5 \\
\hline 42 & Evitar que los demás se encariñen conmigo & 1 & 2 & 3 & 4 & 5 \\
\hline 43 & Tener imágenes de lo que ocurrió & 1 & 2 & 3 & 4 & 5 \\
\hline 44 & No poder pensar en ello por el dolor de cabeza que me produce & 1 & 2 & 3 & 4 & 5 \\
\hline 45 & No esperar tener un trabajo fijo en un futuro & 1 & 2 & 3 & 4 & 5 \\
\hline 46 & Dejar de disfrutar actividades & 1 & 2 & 3 & 4 & 5 \\
\hline 47 & Sensibilidad ante lo que sucede alrededor & 1 & 2 & 3 & 4 & 5 \\
\hline 48 & Intentar no sentir nada cuando hablan de ello & 1 & 2 & 3 & 4 & 5 \\
\hline 49 & Evitar salir de casa por temor a que vuelva a ocurrirme & 1 & 2 & 3 & 4 & 5 \\
\hline 50 & Sentirme solo & 1 & 2 & 3 & 4 & 5 \\
\hline 51 & $\begin{array}{l}\text { Dejar de distinguir entre los sentimientos de alegría o tristeza, como si te fuera indiferente lo que pasa } \\
\text { alrededor }\end{array}$ & 1 & 2 & 3 & 4 & 5 \\
\hline 52 & Enfermarme por el miedo excesivo que me produce & 1 & 2 & 3 & 4 & 5 \\
\hline 53 & Sentir que en cualquier momento volverá a ocurrir & 1 & 2 & 3 & 4 & 5 \\
\hline 54 & Pensar en ello aunque esté con personas que me quieren & 1 & 2 & 3 & 4 & 5 \\
\hline 55 & Tener la sensación de que lo estoy viviendo nuevamente & 1 & 2 & 3 & 4 & 5 \\
\hline 56 & No poder recordar aspectos específicos & 1 & 2 & 3 & 4 & 5 \\
\hline 57 & Tener sensaciones desagradables en el cuerpo cuando observo algo parecido & 1 & 2 & 3 & 4 & 5 \\
\hline 58 & Dejar de importarme tener amigos & 1 & 2 & 3 & 4 & 5 \\
\hline 59 & Tener sueños relacionados & 1 & 2 & 3 & 4 & 5 \\
\hline 60 & No volver a expresar los sentimientos que tuve cuando pasó & 1 & 2 & 3 & 4 & 5 \\
\hline 61 & Responder rápidamente a las agresiones de los demás & 1 & 2 & 3 & 4 & 5 \\
\hline 62 & No conciliar el sueño por sobresaltos & 1 & 2 & 3 & 4 & 5 \\
\hline 63 & Distraerme en clase por estar pensando en lo que pasó & 1 & 2 & 3 & 4 & 5 \\
\hline 64 & Creerme incapaz de formar una familia & 1 & 2 & 3 & 4 & 5 \\
\hline 65 & Evitar tener mejores amigos & 1 & 2 & 3 & 4 & 5 \\
\hline
\end{tabular}

Gracias por tu colaboración 